\title{
LV. The decay of waves in a canal
}

\section{W.J. Harrison B.A.}

To cite this article: W.J. Harrison B.A. (1909) LV. The decay of waves in a canal , Philosophical Magazine Series 6, 18:106, 483-491, DOI: 10.1080/14786441008636723

To link to this article: http://dx.doi.org/10.1080/14786441008636723

册 Published online: 21 Apr 2009.

Submit your article to this journal 준

LII Article views: 3

Q View related articles $₫$ 
(9) The "electric absorption" of gas is found to be closely connected with the size of the cathode fall, which leads to the assumption that the absorption is chiefly caused by positive ions being shot into the cathode.

In conclusion, I wish to thank Sir J. J. Thomson for suggesting the work to me.

I have also much pleasure in thanking $\mathrm{Mr}$. E. Everett for his assistance with some of the glasswork.

Carendish Laboratory,

May 1st, 1909.

LV. The Decay of Waves in a Canal. By W. J. HARRIsos, B.A., Fellow of Clare College, Isaac Nevton Student in the University of Cambridge* .

$\S 1$. TN a paper published recently Dr. R. A. Houstoun gave the results of some experiments on the damping of long waves in a rectangular trough $\uparrow$. The observed damping is from two to three times as great as that calculated from the formula obtained by him. In this paper an explanation of the discrepancy is attempted. It has been shown by the present author that under certain circumstances the air exerts a great damping influence on water waves $\$$. It may be as well to emphasize here the cause of this influence, as it might appear strange that a fluid of such slight density as air could affect the decay of water waves. The modulus of decay $\tau$ of wave-motion at the surface of a single fluid of depth large compared with the wave-length $\lambda$ of the motion depends on $\lambda^{2}$; when there is another fluid superposed $\tau$ depends on $\lambda^{\frac{5}{4}}$; and thus when $\lambda$ is large the difference is considerable, despite the small density of air. Now the waves considered by Dr. Houstoun in his paper are of long wave-length, and it was supposed that the air might have some considerable effect. But it might bave been surmised a priori that this would not be the case, as the effect of the finite depth is itself to produce a first approximation of the order $\lambda^{\frac{5}{4}}$. On obtaining the analytical expression for the damping due to the air, there was found to be not the slightest ground for supposing that we have here the explanation of the discrepancy. Although a consideration of the influence of the air is of no use in our present investigation, the expressions for the

* Communicated by the Author.

+ Phil. Mag. [6] vol. xvii. pp. 154-164.

\$ Proc. Lond. Nath. Scc. Ser. 2, vol. vi. p. 396. 
period and modulus of decay will be given below $\S 3$, on account of their intrinsic interest, and, also, in order that they may be recorded on account of the possibility of their usefulness in some other problem.

It is believed that the real cause of the discrepancr must be looked for along the following lines. Dr. Houstoun in his paper treats of waves of the type $\sin q x \sin r y$, the axes of $x$ and $y$ being horizontal, but his analysis is only suitable for waves of the type sin $q x$. This is clearly brought into evidence by the fact that he obtains for the period of his waves to a first approximation the expression $2 \pi /\left\{g q^{2} h\right\}^{\frac{1}{2}}$, whereas the correct expression is $2 \pi /\left\{g\left(q^{2}+r^{2}\right) h\right\}^{\frac{1}{2}}$, as will be shown later. The same applies to the modulus of decay; he obtains it as a function of $q^{2}$, whereas it is the same function of $\left(q^{2}+r^{2}\right)$. But as will be seen later, this in itself does not wholly explain the discrepancy. It will be shown that we cannot adequately take account of the influence of the sides of the trough in damping the motion. Further discussion will be deferred till the question has been analytically considered.

The damping of long waves at the surface of a single liquid.

\$2. This problem was considered by Hough *, and he obtained an expression for the modulus of decay $\tau$, which is equivalent to

$$
\tau^{-1}=2 k^{2} \nu+\frac{g^{\frac{1}{4}} k^{\frac{5}{4}} \nu^{\frac{1}{2}}}{2 \sqrt{2} \cosh \frac{5}{7} k h \sinh ^{\frac{3}{4}}-k h},
$$

where $v$ is the kinematical coefficient of viscosity, $k=2 \pi / \lambda$, $\lambda$ being the wave-length, and $h$ is the depth of the liquid. The motion under consideration is two-dimensional. The term depending on $v$ in the above expression is not correct; it should be

$$
k^{2} v\left[2+\frac{\cosh ^{2} k h+\sinh ^{2} h h}{4 \cosh ^{2} k h \sinh ^{2} k h}\right] .
$$

This makes an appreciable difference in the case of long waves, since we now have

$$
\frac{1}{\tau}=\frac{\nu}{4 h^{2}}+\left\{\begin{array}{l}
g^{\frac{1}{2}} k \nu \\
8 h^{3}
\end{array}\right\}
$$

This additional term $v / 4 h^{2}$ makes a difference of from 6 to 10 per cent. in the theoretical damping of waves such as those

* Proc. Lond. Math. Soc. vol. xxviii. p. 276 (1897). 
considered by Dr. Houstoun. This will be seen by comparing the third column in Table II. with the sixth column in the table on page 159 of his paper.

The period equation for this motion is given by Bassett *. For convenience it will be repeated here in the notation of this paper.

$$
\left|\begin{array}{ccccc}
m \cosh m h, & -\sinh m h, & 0 & , & 2 v k m a \\
-m \sinh m h, & \cosh m h, & a+2 k^{2} v, & g k \\
-k \sinh k h, & \cosh k h, & 2 k^{2} \nu & , & g k \\
k \cosh k h, & -\sinh k h, & 0 & , & a^{2}+2 v k^{2} a
\end{array}\right|=0,
$$

As regards $i$ and $t$ the motion is of the type $e^{i k x+a t}$.

In the approximation it is assumed that $m h$ is large, on account of the smallness of $v$, and with this assumption the equation becomes

$$
\begin{gathered}
m\left\{a^{2} \cosh k h+g k \sinh k h\right\}-k\left\{a^{2} \sinh k h+g k \cosh k h\right\} \\
+4 k^{2} \nu m a \cosh k h=0 .
\end{gathered}
$$

We obtain by successive approximations

$$
\begin{aligned}
a & = \pm\{g k \tanh k h\}^{\frac{1}{2}}-\frac{1 \pm i}{2 \sqrt{ } 2} \frac{g^{\frac{1}{4}} k^{\frac{5}{2}} \nu^{\frac{1}{2}}}{\cosh ^{\frac{5}{4}} k h \sinh ^{\frac{3}{4}} k h} \\
& -2 k^{2} y-\frac{h^{2} \nu\left(\cosh ^{2} k h+\sinh ^{2} k h\right)}{4 \sinh ^{2} k h \cosh ^{2} k h} .
\end{aligned}
$$

The additional term obtained by this more rigorous method of approximation is negligible compared with $2 k^{2} v$ when the wave-length is small; it has only an appreciable value when the wave-length is large compared with the depth. This is probably the reason why it was omitted from Hough's approximation.

\section{The influence of a superposed fluid on the damping of long waves.}

\$3. The results given in this paragraph have little bearing on the problem under consideration for reasons already given, but it may be as well to record the solution of the period equation to a second approximation.

\footnotetext{
* Treatise on Hydrodynamics, rol. ii. p. 314.
} 
The pericd of the motion is $2 \pi /(\beta-\gamma)$, and the modulus of decay is $\gamma^{-1}$, where

$$
\begin{gathered}
\beta=\left\{\frac{g k\left(\rho-\rho^{\prime}\right) \sinh k h}{\rho \cosh k h+\rho^{\prime} \sinh k h}\right\}^{\frac{3}{2}}, \\
\gamma=\frac{g^{\frac{1}{4}}\left(\rho-\rho^{\prime}\right)^{\frac{1}{4}} k^{\frac{5}{4}} \rho}{2 \sqrt{ } \mathcal{L}\left\{\rho \cosh k h+\rho^{\prime} \sinh k h\right\}^{\frac{5}{4}} \sinh ^{\frac{3}{4}} k h\left(\rho \sqrt{ } \nu^{\prime}+\rho^{\prime} \sqrt{ } \nu\right)} \cdot Q,
\end{gathered}
$$

where

$\mathrm{Q}=\rho\left\{v v^{\prime}\right\}^{\frac{1}{2}}+2 \rho^{\prime} v \cosh h^{2} k h+\rho^{\prime}\left(\nu+v^{\prime}\right) \sinh k h \cosh k h$

$$
+\rho^{\prime}\left(v^{\prime}-v\right) \sinh ^{2} k h \text {; }
$$

$\rho$ and $v$ refer to the lower fluid of depth $h ; \rho^{\prime}$ and $v^{\prime}$ to the superposed fluid.

I have calculated the solution to a third approximation, but the result is too complicated to be given here, and the additional effect derived from it is negligible, even when the second approximation gives an appreciable additional effect.

Some idea of the damping due to air can be derived from a comparison of Tables II. and III.

Waves in a rectangular canal.

\$4. There is one difficulty which Dr. Houstoun escapes by his use of long waves ; it is that the lapping at the sides of the box is virtually neglected, since the vertical motion is not considered. If the problem be attacked in the ordinary way, and the usual conditions of no motion at a fixed boundary be written down, it will be found that the equations are only satisfied by a state of no motion everywhere. The same applies to motion in which slipping at the boundary is allowed, but which is resisted by a traction proportional to the velocity. Hence to discuss the problem at all we are forced to adopt the assumption of a canal with smooth sides. The only alternative is to generalize the method for long waves.

Suppose the canal to be of depth $h$ and breadth $b$. Take the origin of coordinates in the undisturbed surface at one side, the axis of $z$ perpendicular to the side and in the surface, and the axis of $y$ vertically upwards.

The equations of motion are

$$
\begin{aligned}
& \frac{\mathrm{D} u}{\mathrm{D} t}=-\frac{1}{\rho} \frac{\partial p}{\partial x}+\nu \nabla^{2} u \\
& \frac{\mathrm{D} v}{\mathrm{D} t}=-\frac{1}{\rho} \frac{\partial p}{\partial y}-g+\nu \nabla^{2} v \\
& \frac{\mathrm{D} v}{\mathrm{D} t}=-\frac{1}{\rho} \frac{\partial p}{\partial z}+\nu \nabla^{2} w
\end{aligned}
$$


where

$$
\frac{\mathrm{D}}{\mathrm{D} t}=\frac{\partial}{\partial t}+u \frac{\partial}{\partial x}+v \frac{\partial}{\partial y}+w \frac{\partial}{\partial z} .
$$

If we neglect the squares of velocities, these equations are satisfied by

$$
\begin{aligned}
& u=\frac{\partial \phi}{\partial x}+\frac{\partial H}{\partial y}-\frac{\partial G}{\partial z}, \\
& v=\frac{\partial \phi}{\partial y}+\frac{\partial F}{\partial z}-\frac{\partial H}{\partial x^{\prime}}, \\
& w=\frac{\partial \phi}{\partial z}+\frac{\partial G}{\partial x}-\frac{\partial F^{\prime}}{\partial y},
\end{aligned}
$$

where

provided

$$
\frac{p}{\rho}=-\frac{\partial \phi}{\partial t}-g \eta
$$

and

$$
\begin{aligned}
\nabla^{2} \phi & =0 \\
\nu \nabla^{2} \mathrm{~F} & =\frac{\partial \mathrm{F}}{\partial t}, \text { \&c. }
\end{aligned}
$$

Consider

$$
\frac{\partial \eta}{\partial t}=r_{y=0}
$$

$$
\begin{aligned}
& \phi=\left(\mathrm{A}_{1} \cosh m y+\mathrm{A}_{2} \sinh m y\right) \cos \frac{n \pi z}{b} e^{i k x+\alpha t}, \\
& \mathrm{~F}=\left(\mathrm{F}_{1} \cosh m^{\prime} y+\mathrm{F}_{2} \sinh m^{\prime} y\right) \sin \frac{n \pi z}{b} e^{i k x+a t}, \\
& \mathrm{G}=\left(\mathrm{G}_{1} \cosh m^{\prime} y+\mathrm{G}_{2} \sinh m^{\prime} y\right) \sin \frac{n \pi z}{b} e^{i k x+a t}, \\
& \mathrm{H}=\left(\mathrm{H}_{1} \cosh m^{\prime} y+\mathrm{H}_{2} \sinh m^{\prime} y\right) \cos \frac{n \pi x}{b} e^{i k x+a t},
\end{aligned}
$$

where

$$
\begin{aligned}
m^{2} & =k^{2}+n^{2} \pi^{2} / b^{2}, \\
m^{2} & =k^{2}+n^{2} \pi^{2} / b^{2}+a / v \\
& =m^{2}+a / v .
\end{aligned}
$$

These are not the most general expressions of the type which might have been written down, but they are the only ones which satisfy the conditions of the problem. 
The conditions are

(1) $u=v=w=0 \quad(y=-h)$,

(2) $v=0 \quad(z=0, z=b)$,

(3) $\frac{\partial u}{\partial z}+\frac{\partial v}{\partial v}=0, \quad \frac{\partial v}{\partial z}+\frac{\partial w}{\partial y}=0 \quad(z=0, z=b)$,

(4) $\frac{\partial u}{\partial y}+\frac{\partial v}{\partial x}=0, \quad \frac{\partial v}{\partial y}+\frac{\partial v}{\partial z}=0 \quad(y=0)$,

(5) $\frac{\partial \phi}{\partial t}+g \eta+2 v \frac{\partial v}{\partial y}=0 \quad(y=0)$.

Some of these conditions are satisfied identically; from the remainder we derive the six equations

$$
\begin{aligned}
a\left(a+2 v m^{2}\right) \mathrm{A}_{1}+g m \mathrm{~A}_{2}+g \mathrm{U}+2 v m \imath^{\prime} a \mathrm{~V} & =0, \\
2 i k m \mathrm{~A}_{2}+i k \mathrm{U}+m^{\prime} \mathrm{W} & =0, \\
2 m n \pi / b \mathrm{~A}_{2}+n \pi / b \mathrm{U}+m^{\prime} \mathrm{X} & =0,
\end{aligned}
$$

$-m \mathrm{~A}_{1} \sinh m h+m \mathrm{~A}_{2} \cosh m h+\mathrm{U} \cosh m^{\prime} h-\mathrm{V} \sinh m^{\prime} h=0$,

$i k \mathrm{~A}_{1} \cosh m / l-i k \mathrm{~A}_{2} \sinh m h-\mathrm{W} \sinh m^{\prime} h+\mathrm{Y} \cosh m^{\prime} h=0$,

$-n \pi / b \mathrm{~A}_{1} \cosh m h+n \pi / b \mathrm{~A}_{2} \sinh m h+\mathrm{X} \sinh m^{\prime} h-\mathrm{Z} \cosh m^{\prime} h=0$, where

$$
\begin{aligned}
& \mathrm{U}=n \pi / b \mathrm{~F}_{1}-i k \mathrm{H}_{1}, \\
& \mathrm{~V}=n \pi / b \mathrm{~F}_{2}-i k \mathrm{H}_{1}, \\
& \mathrm{~W}=m^{\prime} \mathrm{H}_{1}-n \pi / b \mathrm{G}_{2}, \\
& \mathrm{X}=m^{\prime} \mathrm{F}_{2}-i k \mathrm{G}_{2}, \\
& \mathrm{Y}=m^{\prime} \mathrm{H}_{2}-n \pi / b \mathrm{G}_{1}, \\
& \mathrm{Z}=m^{\prime} \mathrm{F}_{2}-i k: \mathrm{G}_{1} .
\end{aligned}
$$

Among these six quantities there exist the two relations

$$
\begin{gathered}
m^{\prime} \mathrm{U}+i k \mathrm{~W}-n \pi / b \mathrm{X}=0, \\
m^{\prime} \mathrm{V}+i k \mathrm{Y}-n \pi / b \mathrm{Z}=0 .
\end{gathered}
$$

From equations (1) to (8) we eliminate $A_{1}, A_{2}, U, V, W$, $\mathrm{X}, \mathrm{Y}, \mathrm{Z}$, and obtain the period equation.

Approximating on the assumption that $m^{\prime} h$ is large by reason of the smallness of $\nu$, we find that, if we replace $k$ by $m$ in the results for waves propagated in one direction, we obtain the corresponding solution for this problem. This 
result could doubtless be obtained much more simply by generalizing Dr. Houstoun's method. But Dr. Houstoun, although he allows no motion over the sides of the box, yet obtains the same period and damping as for waves propagated in one direction. Now, in the case of wave-motion in the rectangular trough which he used, $m^{2}=\frac{p^{2} \pi^{2}}{a^{2}}+\frac{q^{2} \pi^{2}}{b^{2}}$, for waves of the type $\sin \frac{p \pi x}{a} \sin \frac{q \pi y}{b}$, where $a=152 \cdot 4 \mathrm{cms}$, $b=20.3 \mathrm{cms}$. Hence, for the mode $p=1, q=1$ which he takes as the fundamental one in his solution, $m^{2}=\pi^{2} / b^{2}$, approximately. This leads to quite different results.

The Table below gives the periods of the different modes and for the same depths as given by Dr. Houstoun.

\section{TABLE I.}

\begin{tabular}{|c|c|c|c|c|c|c|}
\hline $\begin{array}{l}\text { Depth } \\
\text { in ems. }\end{array}$ & $\begin{array}{c}\text { Observed } \\
\text { period. }\end{array}$ & $\begin{array}{l}p=1, * \\
q=0 .\end{array}$ & $\begin{array}{l}p=0, \\
q=1 .\end{array}$ & $\begin{array}{l}p=1, \\
q=1 .\end{array}$ & $\begin{array}{c}p=3, \\
q=1 .\end{array}$ & $\begin{aligned} p & =1, \\
q & =3 .\end{aligned}$ \\
\hline 1 & $\begin{array}{l}\text { secs. } \\
10^{*} 1\end{array}$ & $\begin{array}{l}\text { secs. } \\
10.25\end{array}$ & $\begin{array}{l}\text { secs. } \\
1323\end{array}$ & $\begin{array}{l}\text { secs. } \\
1 \cdot 310\end{array}$ & $\begin{array}{l}\text { secs. } \\
1 \cdot 229\end{array}$ & $\begin{array}{l}\text { secs. } \\
\cdot 451\end{array}$ \\
\hline 2 & 5.12 & $7 \cdot 02$ & .938 & .929 & $\cdot 872$ & $\cdot 345$ \\
\hline 3 & 5.80 & $5 \cdot 68$ & $\cdot 779$ & $\cdot 771$ & 725 & $\cdot 313$ \\
\hline 5 & $4 \cdot 49$ & $4 \cdot 39$ & 634 & 629 & $\cdot 596$ & $\cdot 297$ \\
\hline 7 & $3 \cdot 68$ & $3 \cdot 71$ & .573 & $\cdot 569$ & $\cdot 549$ & $\cdot 295$ \\
\hline 10 & $3 \cdot 12$ & $3 \cdot 11$ & 534 & .531 & .509 & $\cdot 294$ \\
\hline
\end{tabular}

In this table the observed periods are those given on page 159 of Dr. Houstoun's paper; it will be noticed that they are slightly different from those on page 161, but the difference is quite immaterial for our purpose.

Thus there can be no doubt that in the motion experimentally observed by Dr. Houstoun there existed the mode $p=1, q=0$. The periods of the other modes are so much shorter that they probably passed unnoticed.

Now, if there is no slipping at the sides and there are no surfaces of slip in the liquid, this mode cannot enter into the motion. But it is evident from the fact that the water rises

* This difference between the quantities in this column and the sum of those in the 3rd and 5th columns on p. 159 of Houstoun's paper is due partly to the fact that the quantities in the 5th column are sometimes miscaleulated.

Phil. Mag. S. 6. Vol. 18. No. 106. Oct. 1909. 2 L 
and falls at the sides that there must be either slipping at the sides, or else there must exist surfaces of slip near the sides in the liquid, most probably both coexist. This fact shows that we have been unable to analyse the motion correctly. The sides must influence the damping of the motion in any actual case, and this has been neglected in the analysis, the sides having been taken as smooth. It will be seen from what has been said above, that we do not know sufficient about the modes in which the water actually vibrates to say that one predominates to such an extent that the damping calculated for that mode should agree with the observed (cf. page 158 of Dr. Houstoun's paper). But even if we admit that part of the observed damping may be due to the existence of higher modes of appreciable amplitude, we have only found an explanation in the case of the depths 1,2 , $3 \mathrm{cms}$. The discrepancy is still unexplained for the depths $5,7,10 \mathrm{cms}$., as will be seen from Table II. Hence the only rational assumption is that the increase of damping is due to the sides. The existence after a time of a surface film such as suggested by Dr. Houstoun would only increase the damping over those values tabulated by him (compare data on pages 159 and 160 of his paper).

Table II. exhibits the reciprocal $(\kappa)$ of the modulus of decay for the same cases as Table I. gives the periods.

TABLE II.

\begin{tabular}{|c|c|c|c|c|c|c|}
\hline $\begin{array}{c}\text { Depth } \\
\text { in cms. }\end{array}$ & $\begin{array}{c}\text { Obserred } \\
\kappa .\end{array}$ & $\begin{array}{c}p=1, \\
q=0 .\end{array}$ & $\begin{array}{c}p=0, \\
q=1 .\end{array}$ & $\begin{array}{c}p=1, \\
q=1 .\end{array}$ & $\begin{array}{c}p=3, \\
q=1 .\end{array}$ & $\begin{array}{c}p=1, \\
q=3 .\end{array}$ \\
\hline 1 & .048 & .0324 & .0826 & .0849 & .0853 & $\cdot 1271$ \\
$\mathbf{2}$ & .032 & .0183 & .0481 & .0463 & .0472 & .0521 \\
3 & .028 & .0133 & .0309 & .0313 & .0316 & .0239 \\
5 & .023 & .0090 & .0165 & .0168 & .0163 & .0075 \\
7 & .022 & .0070 & .0093 & .0093 & .0089 & .0052 \\
10 & .011 & .0053 & .0040 & .0040 & .0037 & .0047 \\
\hline
\end{tabular}

With the last row in this table may be compared the values of $\kappa$ when the damping effect of air is taken into account. These quantities are calculated on the assumption that in the formula in $\$ 3$, we may replace $k$ by $m$, as for waves in a single liquid. 
TABLE III.

\begin{tabular}{|l|l|l|l|l|l|l|}
\hline 10 & .011 & 0053 & .0042 & .0042 & .0039 & .0055 \\
\hline
\end{tabular}

The quantities in Table II. seem to exhibit no regular increase or decrease from one column to another, but this is due to the conflict between the decay due to the internal friction and the decay due to the bottom of the trough.

Clare College,

Cambridge.

IVI. Some Relations in Capillarity. By R. D. KLeeman, D.Sc., B.A., Research Student of Emmanuel College, Cambridge, and McKinnon Student of the Royal Society*.

7 HE potential energy of the surface of a liquid due to the attractions between its molecules has been calculated in general terms by Einstein. Einstein's line of reasoning is as follows. The potential energy of a mass of liquid is first considered. It is assumed that the potential energy of two molecules is given by the expression: $\mathrm{P}_{\infty}-c_{1} c_{2} \psi(r)$ where $\psi(r)$ is some function of the distance between the molecules and $c_{1}$ and $c_{2}$ are constants depending upon the molecules. For $n$ molecules the second term is replaced by

$$
\frac{1}{2} \sum_{a=1}^{a=n} \sum_{\beta=1}^{\beta=n} c_{a} c_{\beta} \psi\left(r_{a}, \beta\right)
$$

If the molecules are identical this becomes

$$
\frac{1}{2} c^{2} \sum_{a=1}^{\alpha=n} \sum_{\beta=1}^{\beta=n} \psi\left(r_{\alpha}, \beta\right) \text {. }
$$

It is next assumed that the potential is the same as if the matter were distributed evenly in space. The potential energy may now be written

$$
\mathrm{P}=\mathrm{P}_{\infty}-\frac{1}{2} c^{2} \mathrm{~N}^{2} \iint d \tau d \tau^{\prime} \psi\left(r_{d \tau, d \tau}\right),
$$

where $d \tau$ and $d \tau^{\prime}$ are elements of volume occupied by matter and $N$ is the number of molecules in unit volume. If each molecule consists of several atoms, we may put $c=\Sigma_{c_{a}}$ as is

* Communicated by the Author.

$2 \mathrm{~L} 2$ 